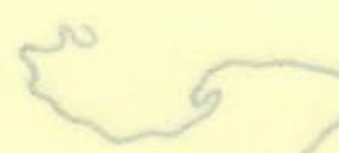

\title{
LA CLASIFICACIÓN DE ESTRUCTURAS DOMÉSTICAS MAYAS UTILIZANDO DATOS DE SUPERFICIE. EL CASO DE EL ROSARIO, CHIAPAS
}

\author{
Olivier DE MonTMOLLIN \\ Universidad de Cambridge \\ Centre of Latin American Studies \\ History Faculty Building
}

Los estudios de patrones de asentamiento, tanto a escala regional como comunitaria, apoyan de modo muy útil el esfuerzo de entender la organización sociopolítica de los mayas de la época clásica. El estudio de patrón de asentamiento que se presenta a continuación es un análisis funcional de datos arquitectónicos de superficie a escala comunitaria. Los datos se obtuvieron en un recorrido intensivo de un sitio del clásico tardío terminal (700-950 d.C.), El Rosario. Este sitio (fig. 1) se encuentra cerca de uno de los altos tributarios del Río Grijalva en Chiapas (Agrinier 1979, 1984; de Montmollin 1979a, 1984). En lo particular, el estudio tiene el fin de determinar cuáles fueron las estructuras "habitacionales" dentro del conjunto de pequeñas estructuras domésticas en El Rosario. Se hace una sencilla distinción funcional entre estructuras "habitacionales" (que sirven para fines generales de vivienda y abrigo para una familia nuclear) y estructuras "adjuntas" (que sirven para propósitos más especiales como almacenamiento, o actividades culinarias, rituales, o manufactureras). Aunque sencillo, tal análisis funcional prepara el terreno para otros estudios de más alcance, tales como estudios acerca de la composición demográfica de unidades domésticas, el ciclo doméstico dentro de las familias (Goody ed. 1962), y el tamaño y la composición demográfica de los barrios y de las comunidades.

Además, un análisis funcional de estructuras domésticas (como éste) tiene un interés metodológico intrínseco, porque 


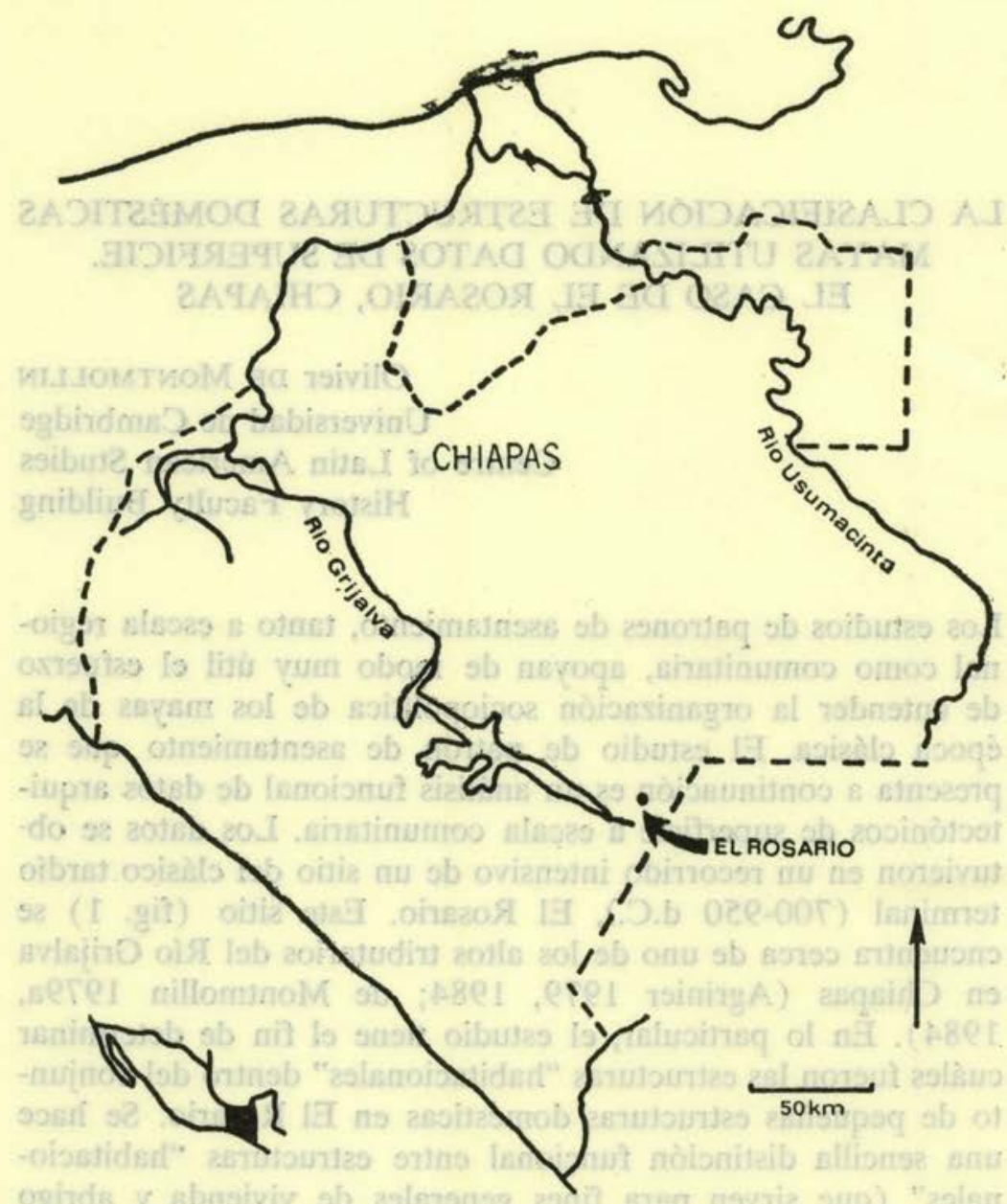

Figura 1. Ubicación de El Rosario.

se trata de sacar el mayor provecho posible de los datos de superficie, un procedimiento muy difícil y lleno de trampas para los incautos. El esfuerzo vale la pena, sin embargo, porque el uso de datos de superficie permite una más amplia cobertura espacial que los estudios que utilizan solamente datos sacados de excavaciones. Más ampliamente, las cuestiones de índole metodológica merecen nuestrạ atención porque el análisis de 
patrones arqueológicos tiene un sinfín de ambigüedades y eso necesita que se preste tanta o mayor atención al modo de llegar a las conclusiones como a las conclusiones mismas.

Antes de emprender un estudio funcional que necesita muchas comparaciones entre estructuras y unidades domésticas dentro de un sitio, es necesario hacerse varias preguntas acerca de la calidad de los datos disponibles para averiguar si tales datos de superficie se prestan adecuadamente a este tipo de manejo analítico. Las preguntas giran en torno a la contemporaneidad de las estructuras domésticas (los problemas de control cronológico), la amplitud con que se registraron las estructuras domésticas, y la representatividad de los patrones encontrados (ęn este caso, los patrones en El Rosario con referencia a otros sitios en su valle o en otras partes de la zona maya).

En lo que se refiere a la cuestión cronológica, datos sacados de reconocimientos y excavaciones (Agrinier 1979; de Montmollin 1979a, 1979b, 1984) nos sugieren que el patrón de asentamiento en El Rosario representa a un conjunto de estructuras domésticas utilizadas contemporáneamente durante una sola época -el clásico tardío terminal-. Dicho de otra manera, el mapa de El Rosario" (fig. 2) no muestra una mezcla de asentamientos de varias épocas. Dentro del lapso del clásico tardío terminal, se puede proponer que la gran mayoría de las estructuras domésticas (y ceremoniales) se utilizaron contemporáneamente durante un periodo de auge, inmediatamente antes de que El Rosario dejara de ser un importante asentamiento. Los datos que apoyan tal aseveración se han expuesto en otros trabajos (de Montmollin 1984, 1985a). Aquí, me limito a notar que tal interpretación de la cronología del asentamiento se asemeja de modo general a las interpretaciones de otros mayistas acerca del lapso hacia fines de la época clásica (ver Ashmore ed. 1981) y se basa en el fenómeno del colapso maya, un colapso que tocó también a El Rosario y su zona circundante.

Otro problema que asoma es el de saber si se registraron todas las estructuras domésticas presentes en el sitio y no solamente las menos perecederas o las menos visibles. ¿Hasta qué grado serán los espacios blancos en el mapa producto de la técnica (y de las condiciones) de investigación o resultado de la verdadera distribución de estructuras domésticas? Dado 


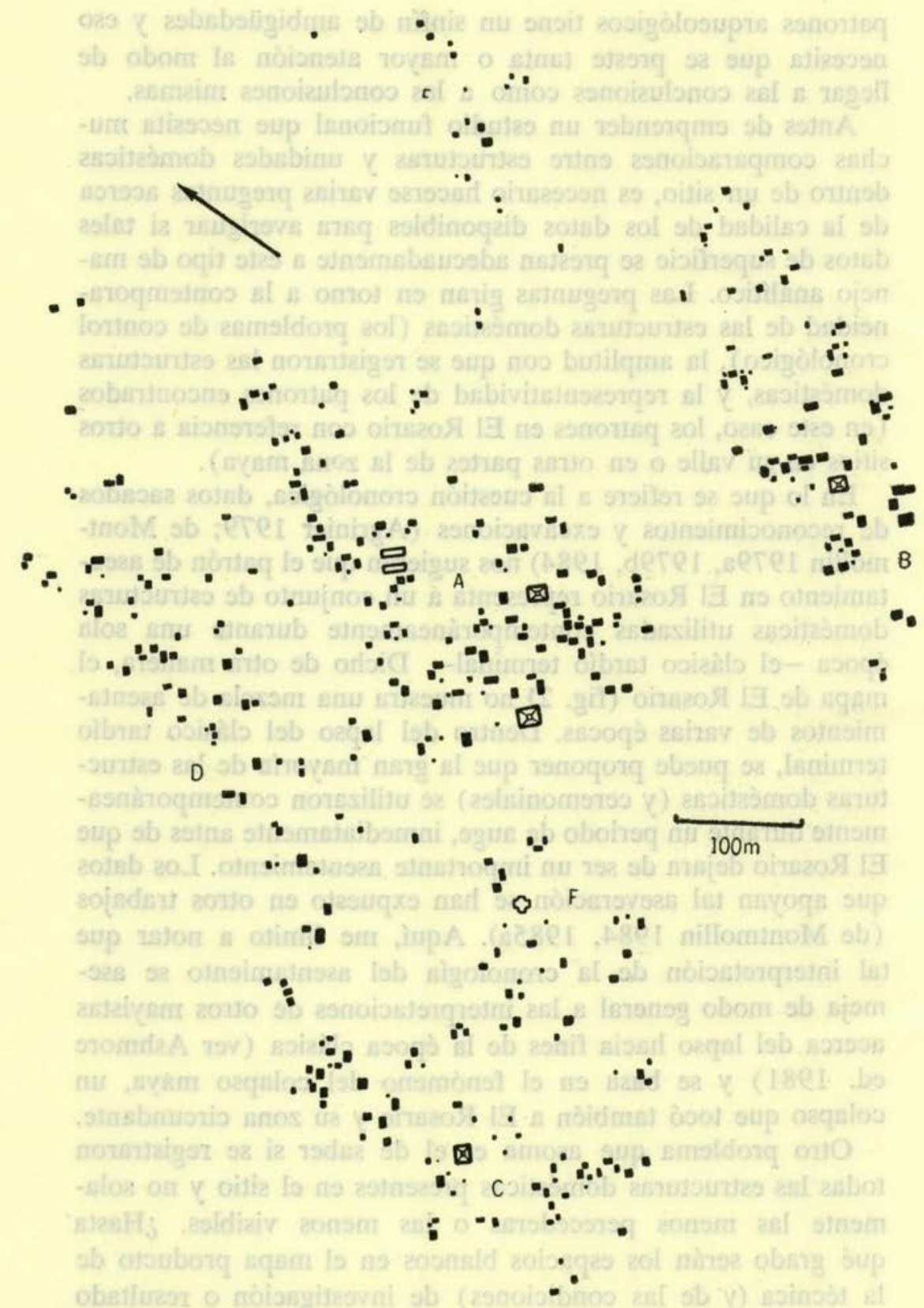

Figura 2. El Rosario. 
lo poco hondo de la capa de suelo sobre la zona, y dadas las buenas condiciones de visibilidad arqueológica (con muy escasa vegetación), puedo aseverar que se registró la arrolladora mayoría de las estructuras domésticas con piedra en su sistema constructivo. Estructuras sin piedra no se localizaron, pero es factible suponer que toda estructura con fines habitacionales hubiera necesitado una plataforma con muros de retención de piedra para protegerla de los efectos destructivos del agua. A fin de cuentas, en El Rosario se registraron con suficiente amplitud las estructuras domésticas necesarias para un análisis funcional a escala comunitaria.

La tercera pregunta a hacerse acerca de los datos de superficie tiene que ver con el grado de representatividad, en este caso la representatividad de los patrones de El Rosario, sobre todo en lo que se refiere a otros sitios cercanos (en el valle Santa Inés/El Rosario). El tema tiene importancia porque los resultados del análisis funcional de estructuras domésticas en El Rosario han servido como ayuda para interpretar los patrones de muchos otros sitios registrados en un reconocimiento intensivo del valle Santa Inés/El Rosario (de Montmollin $1985 \mathrm{a}, 1985 \mathrm{~b})$. Entonces, es útil tener una buena idea del lugar que ocupa El Rosario en su marco regional. El tamaño doméstico de El Rosario (300 estructuras "habitacionales") es grande comparado con las normas que rigen en el valle. Las plazas cívico-ceremoniales en El Rosario le dan un rango de tercer orden dentro de la jerarquía política del valle; sirve como centro rector de uno de los "distritos" dentro del reino Rosario (de Montmollin 1985b, 1985c), cuya capital es Tenam Rosario (Agrinier 1983). En lo que se refiere al tamaño y arreglo de sus unidades domésticas, y al tamaño y la forma de sus estructuras domésticas individuales, El Rosario se parece mucho a los otros sitios en el valle. Entre paréntesis, se puede notar que en general el tamaño de las estructuras domésticas en el valle Santa Inés/El Rosario (que típicamente sería de unos $6 \times$ $4 \mathrm{~m}$ ) se encuentran al extremo "pequeño" de la gama de tamaños dentro de la zona maya, al igual que las estructuras domésticas del norte de Yucatán (Tourtellot 1983: 37-38; Kurjack 1974). Además, las estructuras domésticas en El Rosario no difieren de otras en el valle en su orientación, material constructivo, o estilo de construcción. Resumiendo, los patrones de asen- 
tamiento entre las unidades y estructuras domésticas en El Rosario son bastante representativos para que se puedan extender los resultados de un análisis funcional en este sitio a otros sitios del clásico tardío terminal en el valle Santa Inés/El Rosario. Como tipología de estructuras domésticas, durante la recolección de datos, se utilizó una Clasificación Funcional Provisional [o CFP] con dos grandes categorías: 1. estructuras habitacionales y 2. estructuras adjuntas. Entre las últimas hay: 2A. estructuras sub-habitacionales (por su tamaño), y 2B. altares rituales. Hacer la distinción entre estructuras habitacionales y altares no era difícil ya que los últimos tienen rasgos muy claros de tamaño (casi nunca rebasan los $2.5 \times 2.5 \mathrm{~m}$ ) y de localización (en el lado "abierto" de su unidad doméstica). Para abarcar el problema más difícil de distinguir entre estructuras habitacionales y subhabitacionales se utilizaron criterios de forma arquitectónica - las dimensiones de la plataforma- (lo que equivale al piso) y por extensión su área. Para las estructuras habitacionales, la plataforma debía tener un eje corto con al menos $3.0 \mathrm{~m}$ y un mínimo de $9.0 \mathrm{~m}^{2}$ (o en casos especiales, un eje corto de al menos $2.0 \mathrm{~m}$, con el eje largo de al menos $4.0 \mathrm{~m}$, y asî un mínimo de $8.1 \mathrm{~m}^{2}$ ). Para las estructuras adjuntas subhabitacionales, la plataforma debía tener un eje corto con más de $2.0 \mathrm{~m}$ y menos de $3.0 \mathrm{~m}$ y un eje largo de menos de $3.5 \mathrm{~m}$, dando un máximo de $10.4 \mathrm{~m}^{2}$ (o en casos especiales, un eje corto de no más de $2.0 \mathrm{~m}$, un eje largo de menos de $3.0 \mathrm{~m}$, y así un máximo de $6.0 \mathrm{~m}^{2}$ ). En la siguiente discusión se refiere a las estructuras habitacionales (definidas según la CFP) como estructuras HAB y se refiere a las estructuras adjuntas subhabitacionales (también definidas según la CFP) como estructuras ADJ.

Los criterios utilizados en la CFP se apoyan en ideas derivadas del "sentido común" acerca de que lo que serían las mínimas dimensiones para los fines habitacionales de una sola familia (nuclear). Estas ideas pueden ser erróneas, pero por el momento no parecen peores que las ideas que se basan en grandes comparaciones transculturales, por ejemplo, la idea de que siempre se necesita un promedio de $10 \mathrm{~m}^{2}$ por persona para fines habitacionales (Naroll 1962; Puleston 1973: 179-185). Pero, además del sentido común, habrá otras maneras de establecer para El Rosario cuáles entre las estructuras HAB sir- 
vieron como viviendas. Las excavaciones que se llevaron a cabo (entre 18 de las 300 estructuras $\mathrm{HAB}$ ) no indican que tales estructuras tuvieron funciones especiales no asociadas con fines generales de vivienda y abrigo (Montmollin 1979a; Agrinier 1979). Sin embargo, este tipo de dato no es satisfactorio porque no tiene directamente que ver con la gran mayoría de estructuras no excavadas. Es más directo tratar de juzgar la validez (o falta de validez) de la CFP refiriéndose a datos de todas las estructuras en El Rosario. La meta principal en este estudio es tratar de averiguar si cada una de las 300 estructuras $\mathrm{HAB}$ era una estructura habitacional o si tal vez había estructuras adjuntas entre el grupo. No se trata aquí de las estructuras ADJ porque son muy pocas (sólo 32) y la cuestión de la exactitud de su definición no afecta tanto la validez global de la CFP.

Vemos ahora a grandes rasgos cuál es el patrón de asentamiento doméstico en El Rosario. Las 300 estructuras HAB se encuentran distribuidas entre unas 163 unidades domésticas (fig. 3 ; cuadro 1). Cada unidad doméstica tiene entre una y siete estructuras $\mathrm{HAB}$ y $\mathrm{ADJ}$ o entre una y cuatro estructuras HAB. Las unidades domésticas se reparten entre seis divisiones dentro del sitio. Estas divisiones pueden asemejarse a barrios y aquí se denominan secciones ( $\mathrm{A} \mathrm{a} \mathrm{F}$, en la fig. 2).

\section{Cuadro 1}

Estructuras $\mathrm{HAB}$ y $\mathrm{ADJ}$ en El Rosario

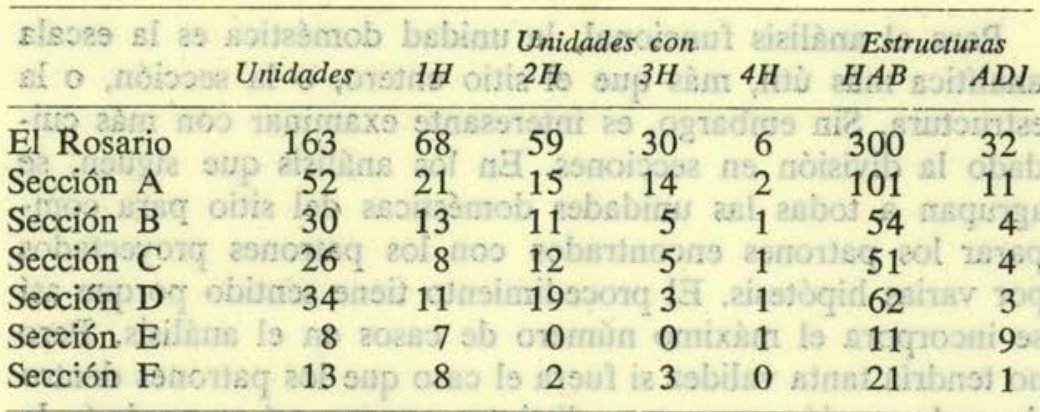

Nota: $H=$ Estructura $\mathrm{HAB}$ (habitacional según la Clasificación Funcional Provisional [CFP]).

$\rightarrow$ ih $\mathrm{ADJ}$ : Estructura adjunta. (según la CFP). 


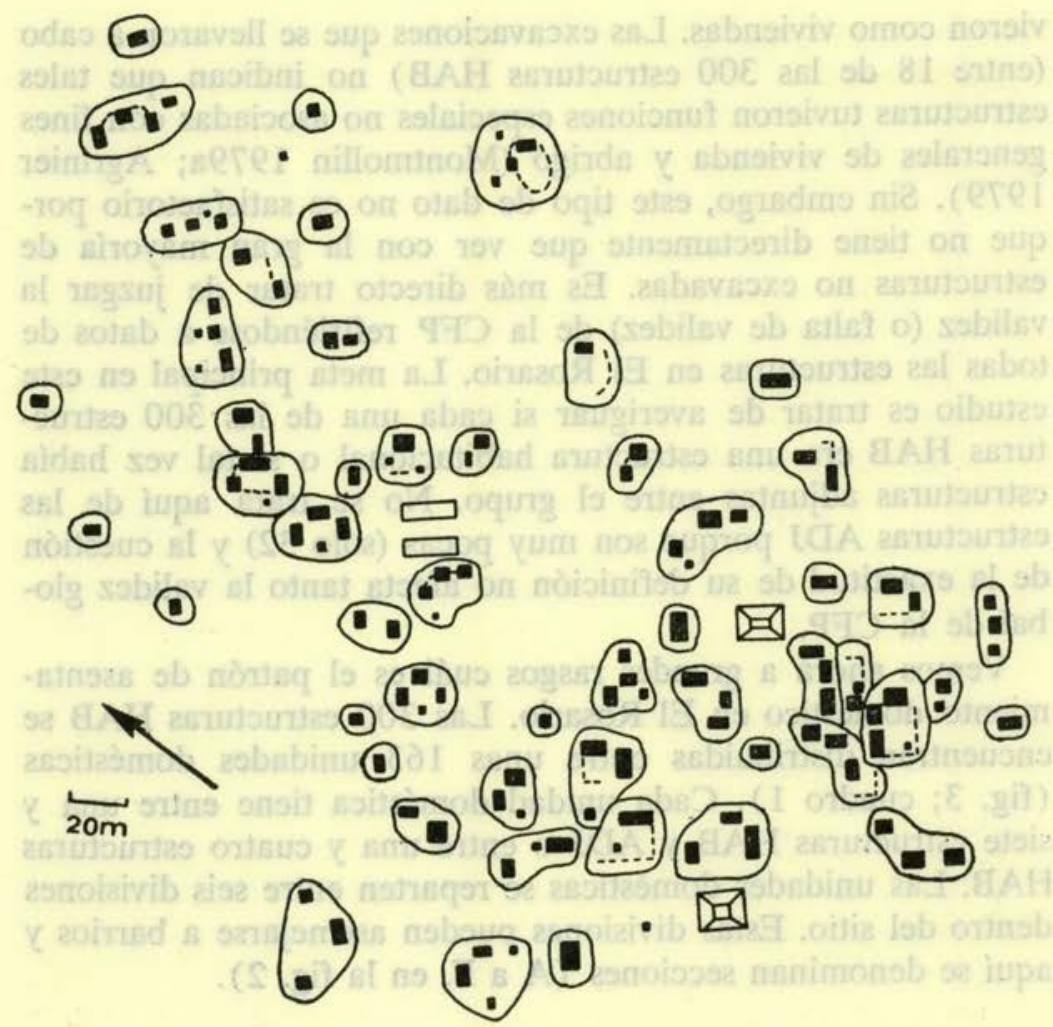

Figura 3. El Rosario, Sección A (con las Unidades Domésticas Delineadas).

Para el análisis funcional, la unidad doméstica es la escala analítica más útil, más que el sitio entero, o la sección, o la estructura. Sin embargo, es interesante examinar con más cuidado la división en secciones. En los análisis que siguen, se agrupan a todas las unidades domésticas del sitio para comparar los patrones encontrados con los patrones proyectados por varias hipótesis. El procedimiento tiene sentido porque así se incorpora el máximo número de casos en el análisis. Pero no tendría tanta validez si fuera el caso que los patrones dentro de cada sección eran muy distintos porque así se perdería la ocasión de estudiar esa variabilidad. Se puede ver que cada una de las variables utilizadas en el análisis tiene valores algo dis- 
tintos para cada sección (cuadros 2 y 3), pero los valores no son suficientemente distintos para distorsionar en gran medida el análisis que trata del sitio entero. Además, cuando se hace una serie de análisis separados para las secciones, se encuentran los mismos patrones y resultados en cada una de las cuatro secciones más grandes (con el $89 \%$ de las unidades domésticas) que se encuentran en el análisis del sitio entero (de Montmollin 1981).

Antes de emprender el análisis funcional necesitamos hacer varias suposiciones: 1 . Se supone que todas las unidades domésticas tienen una función habitacional y por eso deben tener

\section{Cuadro 2}

Áreas de las estructuras $\mathrm{HAB}$ en las secciones de El Rosario $\left(\mathrm{m}^{2}\right)$

\begin{tabular}{crrrrr}
\hline Sección & $n$ & Promedio & Mediana & Minimo & Máximo \\
\hline A & 101 & 27.6 & 28.4 & 9.8 & 57.8 \\
B & 54 & 28.3 & 25.5 & 11.4 & 110.0 \\
C & 51 & 21.9 & 20.0 & 9.2 & 42.7 \\
D & 62 & 25.2 & 22.1 & 9.0 & 65.0 \\
E & 11 & 17.0 & 15.6 & 9.0 & 39.4 \\
F & 21 & 24.2 & 20.7 & 13.6 & 40.3 \\
Todas & 300 & 25.6 & 23.1 & 9.0 & 110.0 \\
\hline
\end{tabular}

\section{Cuadro 3}

Alturas de las estructuras $\mathrm{HAB}$ en las secciones de El Rosario (m)

\begin{tabular}{crcccc}
\hline Sección & $n$ & Promedio & Mediana & Minimo & Máximo \\
\hline A & 101 & 0.64 & 0.60 & 0.15 & 1.26 \\
B & 54 & 0.59 & 0.54 & 0.20 & 1.42 \\
C & 51 & 0.54 & 0.51 & 0.20 & 1.03 \\
D & 62 & 0.57 & 0.51 & 0.22 & 1.40 \\
E & 11 & 0.37 & 0.32 & 0.10 & 0.74 \\
F & 21 & 0.55 & 0.54 & 0.30 & 0.87 \\
Todas & 300 & 0.58 & 0.54 & 0.10 & 1.42 \\
\hline
\end{tabular}


al menos una estructura habitacional. 2. Se supone que todas las estructuras $\mathrm{HAB}$ tienen la posibilidad (o potencialidad) de haber funcionado como estructuras habitacionales. 3. Se supone que cuando hay más de una estructura $\mathrm{HAB}$ dentro de una unidad doméstica, la estructura HAB de mayor tamaño es siempre una estructura habitacional. Las estructuras HAB de menor tamaño tienen más posibilidad de haber funcionado como estructuras adjuntas. Utilizando el primer y tercer supuesto se llega a un total de 163 estructuras HAB cuya función habitacional se acepta de antemano (eso corresponde al número de unidades domésticas registradas). Quedan entonces 137 estructuras $\mathrm{HAB}$ cuya posible función habitacional se debe investigar.

Para tal investigación, es útil ordenar las estructuras HAB dentro de su propia unidad según varios criterios. Esto se relaciona con la lógica del tercer supuesto, de que el tamaño de las estructuras dentro de su unidad tiene que ver con su funciôn. No tiene sentido ordenar las estructuras en todo el sitio (o en toda una sección) porque es probable que la variación entre las unidades puede producir casos en que hay estructuras adjuntas (de unidades con estructuras generalmente grandes) más grandes que ciertas estructuras habitacionales (de unidades con estructuras generalmente pequeñas). Tal variación entre estrueturas y unidades puede esperarse en un sistema con estratificación sociopolítica tal como existía en El Rosario.

Para fines de análisis funcional, se puede examinar la validez de una serie de hipótesis bastante sencillas. La primera, Hipótesis "A", propone que no hubo más de una estructura habitacional dentro de cada unidad doméstica. Utilizando datos de superficie, ¿cómo se puede poner a prueba tal hipótesis? $\mathrm{Si}$ fuera válida, se esperaría encontrar un patrón en que las estructuras adjuntas se encontrarían con estructuras habitacionales de gente de alta categoría sociopolítica. Eso tiene que ser porque tales "estructuras adjuntas" (encontradas en las unidades domésticas con dos a cuatro estructuras HAB, según la Hipótesis "A") se ven bastante caras y ostentosas en comparación con la ausencia de estructuras adjuntas (o tal vez la presencia de estructuras adjuntas perecederas no registradas en el recorrido). Lógicamente, los moradores en las estructuras habitacionales de 
alta categoría sociopolítica tendrían más capacidad económica y suntuaria para construirse estructuras adjuntas relativamente más elaboradas. Se ha razonado de la misma manera con referencia a unidades domésticas en Tikal (Puleston 1973: 129; Haviland 1981: 99). Si eso fuera el caso en El Rosario, al examinar distribuciones de estructuras habitacionales según criterios de rango sociopolítico, se encontraría a distintas distribuciones de estructuras habitacionales con y sin estructuras adjuntas.

¿Cuáles criterios (arquitectónicos) de rango sociopolítico se pueden utilizar? Es una pregunta muy difícil de contestar y que tiene muchas complejidades (ver Blake 1984; Agrinier 1979; de Montmollin 1981). En breve, para los fines de este análisis, se han escogido dos variables como criterios de rango sociopolítico: el área de la plataforma de una estructura, y la altura (promedio) de tal plataforma.

Por sí solo, el área (del piso) no es un criterio idóneo. No es claro si el área refleja factores demográficos (tamaño de la familia) o factores de rango sociopolítico. Si se supone un valor invariable para la cantidad de área por persona en cada sitio, sección, o rango sociopolítico (al estilo de $l o s ~ 10 \mathrm{~m}^{2}$ por persona de Naroll 1962), entonces las variaciones en las áreas representan variaciones demográficas (al momento de construir las plataformas). Alternativamente, si se supone que la cantidad de área por persona puede variar, entonces las variaciones en las áreas pueden representar variaciones sociopolíticas, con las familias de más alto rango teniendo más amplios espacios (per cápita) en sus viviendas. Para confundir más, es posible que las familias de más alto rango tuvieran por lo común más individuos. Tal caso daría un patrón en que las estructuras habitacionales más grandes de alta categoría, y las estructuras habitacionales más pequeñas de baja categoría, tendrían las mismas proporciones de área por persona. Con tantos problemas, ¿por qué escoger el área como criterio de rango sociopolítico? En el caso del valle Santa Inés/El Rosario, eso se puede justificar de manera indirecta con referencia a un grupo de estructuras habitacionales que claramente tienen alta categoría sociopolítica. Éstas son las llamadas "estructuras alargadas" ("range structures" en inglés) que bordean a las plazas cívicas en algunos sitios del valle Santa Inés/El Rosario (aunque no se encuentran en El Rosario mismo). Como se ve por 
su nombre, las estructuras alargadas tienen un eje bastante amplio (entre 15 y 40 m o más) comparado con una largura normal de 4-6 m para la mayoría de las estructuras domésticas. Por fuerza, las estructuras alargadas tienen también un área más grande. Entonces, ese patrón da alguna validez a la idea de que por lo general las estructuras más largas (y con más área) tenían moradores de rango sociopolítico más alto.

La altura de la plataforma es un criterio más claro para juzgar el rango sociopolítico de sus habitantes. No hay modo en que la altura se pueda relacionar con el tamaño de la familia. En cambio, cualquier alzamiento de una plataforma más allá de lo mínimo necesario para proteger los muros y los pisos de los efectos dañinos del agua, corresponde a motivos de ostentación sociopolítica (Agrinier 1979; Kurjack 1974: 51).

En fin, aquí no se utiliza el volumen de la plataforma como criterio de rango sociopolítico porque los resultados son bastante parecidos a los que se logran utilizando el área y la altura de la plataforma. Además, los volúmenes de las plataformas en El Rosario (con un promedio de $17.3 \mathrm{~m}^{3}$ por estructura) no son suficientemente grandes para sobrecargar la fuerza de trabajo de una sola familia. Entonces los métodos que evalúan el rango sociopolítico de las personas y familias, según la inversión de fuerza de trabajo en sus casas (Kurjack 1974; Sanders 1974), no tienen mucha vigencia en este caso.

$\mathrm{Al}$ tener los criterios de rango sociopolítico en la mano, se puede volver a la Hipótesis "A" para ponerla a prueba. Primero, se seleccionan las estructuras $\mathrm{HAB}$ con las áreas más grandes en sú propia unidad doméstica. Después dos distribuciones de tales estructuras se ponen sobre el mismo eje; una para las estructuras que vienen de unidades con otras estructuras $\mathrm{HAB}$ (o con estructuras adjuntas según la hipótesis), y una para las estructuras que vienen de unidades sin más estructuras HAB (o sin estructuras adjuntas según la misma hipótesis). Se sigue el mismo procedimiento con la distribución de las alturas de las estructuras. En ambos casos los resultados son parecidos (figs. 4 y 5). Por lo general las estructuras $\mathrm{HAB}$ con estructuras adjuntas son de mayor tamaño y rango sociopolítico que las que carecen de estructuras adjuntas. Pero las dos distribuciones se traslapan a tal grado (el $29 \%$ de los casos 
Figura 4. Distribuciones de Áreas de Estructuras HAB de Primer Rango con y $\sin$ Estructuras Adjuntas.

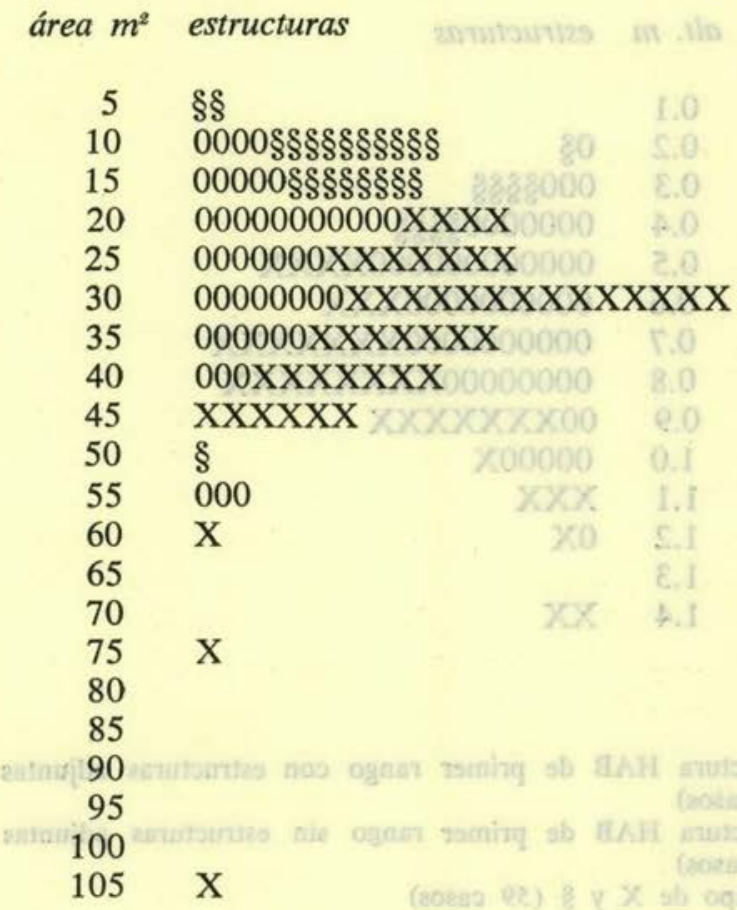

Notas:

$\mathrm{X}=$ estructura HAB de primer rango con estructuras adjuntas (95 casos)

$\S=$ estructura $\mathrm{HAB}$ de primer rango sin estructuras adjuntas (68 casos)

$0=$ traslapo de $X$ y $\$$ (47 casos) (total $=163$ casos).

El área de las plataformas se utiliza de dos modos distintos:

1. El rango del área de una estructura dentro de su unidad doméstica indica su función. Las estructuras de primer rango son estructuras habitacionales y las de rango menor son estructuras adjuntas.

2. La ubicación de una estructura dentro de la distribución global de áreas indica el rango sociopolítico de sus moradores dentro del sitio.

Este doble uso caracteriza también al manejo de la altura de las plataformas (Fig. 5). 
Figura 5. Distribucionés de Alturas de Estructuras HAB de Primer Rango con y $\sin$ Estructuras Adjuntas.

$\begin{array}{ll}\text { alt. } m & \text { estructuras } \\ 0.1 & \\ 0.2 & 0 \S \\ 0.3 & 000 \S \S \S \S \\ 0.4 & 0000000 \S \S \S \S \\ 0.5 & 000000000000 \mathrm{XXXX} \\ 0.6 & 0000000000 \mathrm{XXX} \\ 0.7 & 0000000000 \mathrm{XXXXXXX} \\ 0.8 & 00000000 \mathrm{XXXXXXXX} \\ 0.9 & 00 \mathrm{XXXXXXX} \\ 1.0 & 00000 \mathrm{X} \\ 1.1 & \mathrm{XXX} \\ 1.2 & 0 \mathrm{X} \\ 1.3 & \\ 1.4 & \mathrm{XX}\end{array}$

Notas:

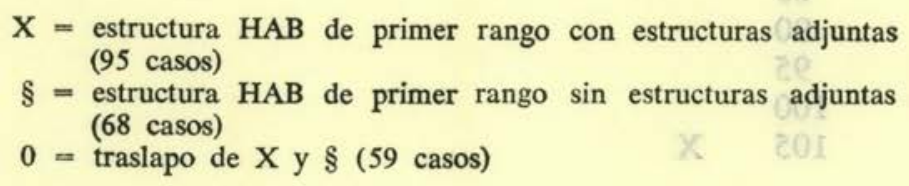

con el área y el 36\% de los casos con la altura) que se puede rechazar la Hipótesis "A" de que había una sola estructura habitacional en cada unidad doméstica de múltiples estructuras. Con estos resultados en la mano, se puede proponer que en El Rosario no había una regla muy estricta de fisión doméstica en la que el crecimiento de la familia siempre tuviera como resultado la fundación de una nueva unidad doméstica. En cambio, parece que el crecimiento familiar podía resultar en la construcción de otras estructuras habitacionales dentro de la misma unidad doméstica.

Siguiendo con la evaluación de la CFP, se puede manejar otra hipótesis, la Hipótesis "B" que propone que había cantidades rariables de estructuras habitacionales dentro de cada unidad toméstica, y que todas las estructuras habitacionales 
dentro de una unidad doméstica tenían el mismo (o casi el mismo) tamaño. Con esta hipótesis se introduce la dificultad de tener que determinar cuál es la diferencia de tamaño suficiente entre las estructuras HAB dentro de una unidad doméstica) para separar las estructuras habitacionales de las estructuras adjuntas. Un poco arbitrariamente, se decidió clasificar como posibles estructuras habitacionales todas las estructuras con un área dentro de $10 \mathrm{~m}^{2}$ del área de la estructura con mayor área en su unidad. La estructura mayor y tales otras estructuras se denominan estructuras "primarias". Las estructuras más pequeñas que la estructura mayor en su unidad por más de $10 \mathrm{~m}^{2}$ se denominan estructuras "secundarias" y tienen más posibilidad de haber sido estructuras adjuntas. Por lo que se refiere a la altura, la estructura más alta y todas las estructuras con una altura dentro de $0.15 \mathrm{~m}$ de su altura se clasificaron como estructuras "primarias" con mayor posibilidad de haber funcionado como estructuras habitacionales. Y todas las estructuras más de $0.15 \mathrm{~m}$ más bajas que la estructura más alta se clasificaron como estructuras "secundarias" con mayor posibilidad de haber sido estructuras adjuntas. Una diferencia de altura de $0.15 \mathrm{~m}$ se escogió con la idea de que ésa corresponde al intervalo mínimo perceptible para una plataforma (una o dos hiladas de piedras para El Rosario).

El procedimiento para poner a prueba la Hipótesis "B" es parecido al que se utilizó para evaluar la Hipótesis "A". Si fuera válida la Hipótesis "B", se esperaría encontrar que las estructuras $\mathrm{HAB}$ con estructuras secundarias en su unidad tuvieran más alta categoría sociopolítica que las estructuras $\mathrm{HAB}$ sin tales estructuras. Además, no se vería mucho traslapo entre las distribuciones de los dos tipos de estructuras HAB sobre un continuo de rango sociopolítico. Utilizando los mismos criterios de rango sociopolítico, se encuentra tal traslapo entre las dos distribuciones $(25 \%$ de los casos con el área y $31 \%$ de los casos con la altura-figs. 6 y 7) que se puede rechazar la hipótesis. Una interpretación de tales resultados es que en El Rosario había a veces diferencias apreciables de status sociopolítico dentro de la misma unidad doméstica. Además, la variabilidad en el número de estructuras habitacionales en las distintas unidades domésticas puede corresponder a los resultados de un ciclo doméstico de la familia nuclear- 
Figura 6. Distribuciones de Áreas de Estructuras HAB Primarias con y sin Estructuras Adjuntas.

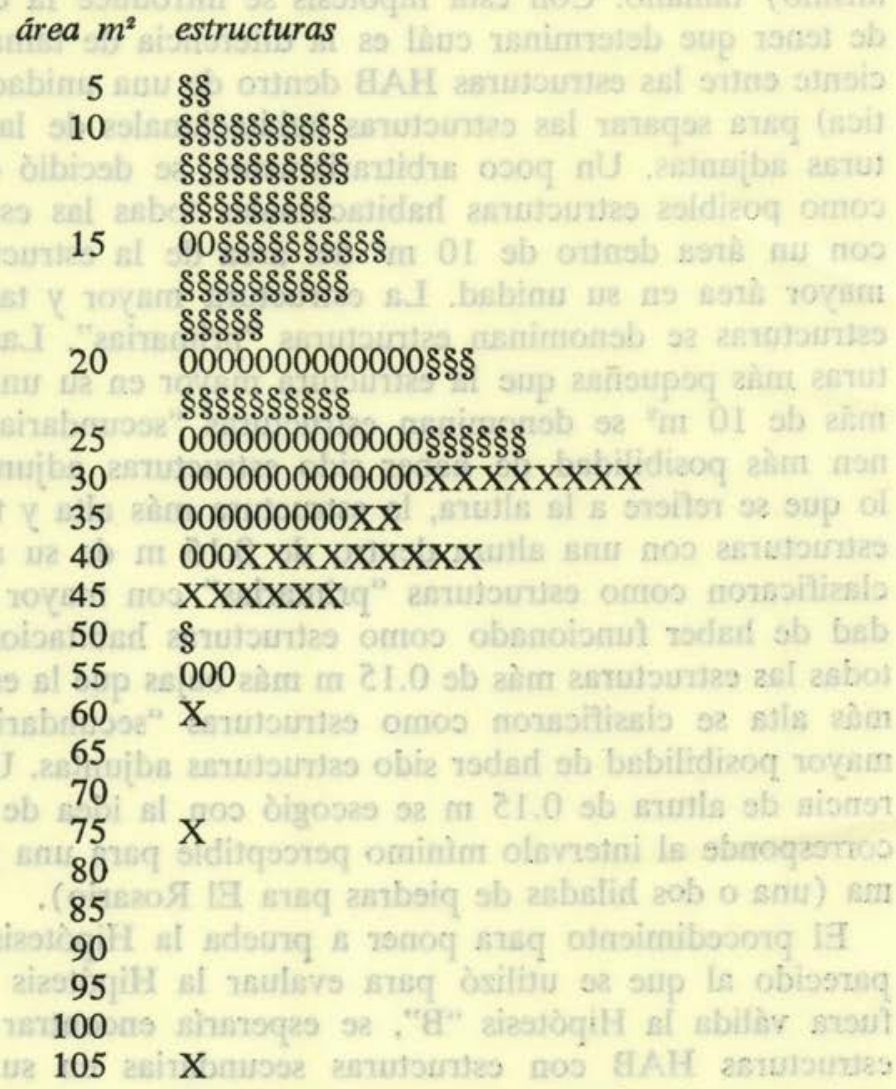

Notas:

$\mathrm{X}=$ estructura $\mathrm{HAB}$ primaria con estructuras adjuntas

(84 casos)

$\S \div$ estructura $\mathrm{HAB}$ primaria sin estruclturas adjuntas (136 casos)

0 = traslapo de $X$ y $\S(56$ casos) (total $=220$ casos).

El área de la plataforma se utiliza de dos modos distintos:

1. El área de una estructura dentro de su unidad doméstica indica su función. Las estructuras primarias son estructuras habitacionales y las secundarias son estructuras adjuntas.

2. La ubicación de una estructura dentro de la distribución global de áreas indica el rango sociopolítico de sus moradores dentro del sitio.

Este doble uso caracteriza también al manejo de la altura de las plataformas (Fig. 7). 
Figura 7. Distribuciones de Alturas de Estructuras HAB Primarias con y sin Estructuras Adjuntas.

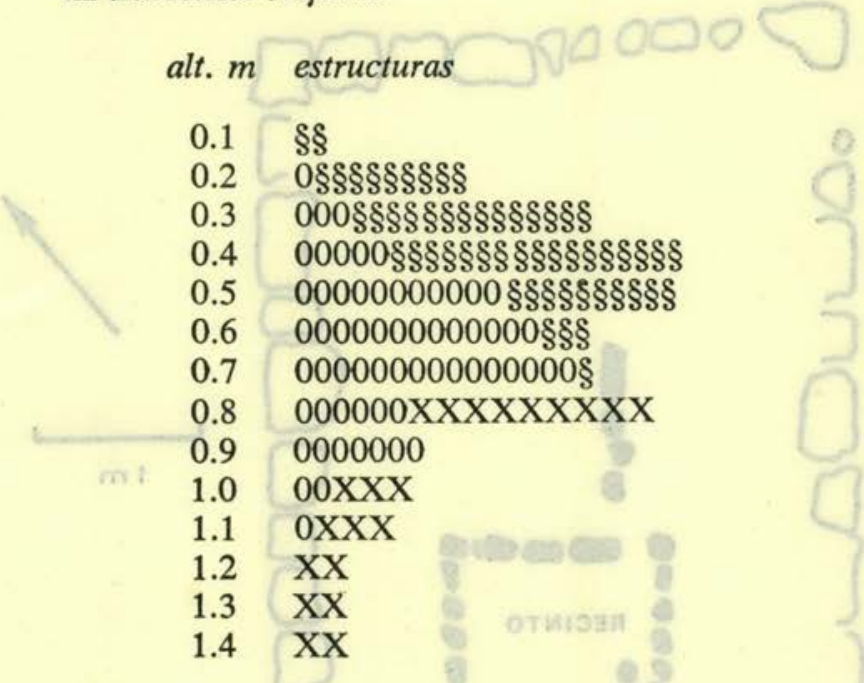

Notas:

$\mathrm{X}=$ estructura $\mathrm{HAB}$ primaria con estructuras adjuntas (84 casos)

$\S=$ estructura $\mathrm{HAB}$ primaria sin estructuras adjuntas (123 casos)

$0=$ traslapo de $\mathrm{X}$ y $\S(64$ casos) (total $=207$ casos).

extensa (Goody ed. 1962; Siverts 1969: 74-78). Según esta hipótesis, los distintos números de estructuras habitacionales en las unidades domésticas representan distintas fases en el ciclo de crecimiento (de Montmollin 1985a; Tourtellot 1983: 48; Folan et al., 1983: 134-135, 140-141).

Para realizar el análisis funcional de estructuras en El Rosario se cuenta con un rasgo más cualitativo de la arquitectura doméstica: la presencia o ausencia de "elementos cuadrangulares" colocados sobre los pisos de las estructuras. Estos elementos cuadrangulares son pequeñas plataformas o pequeños recintos que raramente rebasan en su tamaño los 2 por $2 \mathrm{~m}$, con $10-20 \mathrm{~cm}$ de alto (fig. 8). Se han encontrado elementos parecidos en otros sitios clásicos de los altos tributarios del 


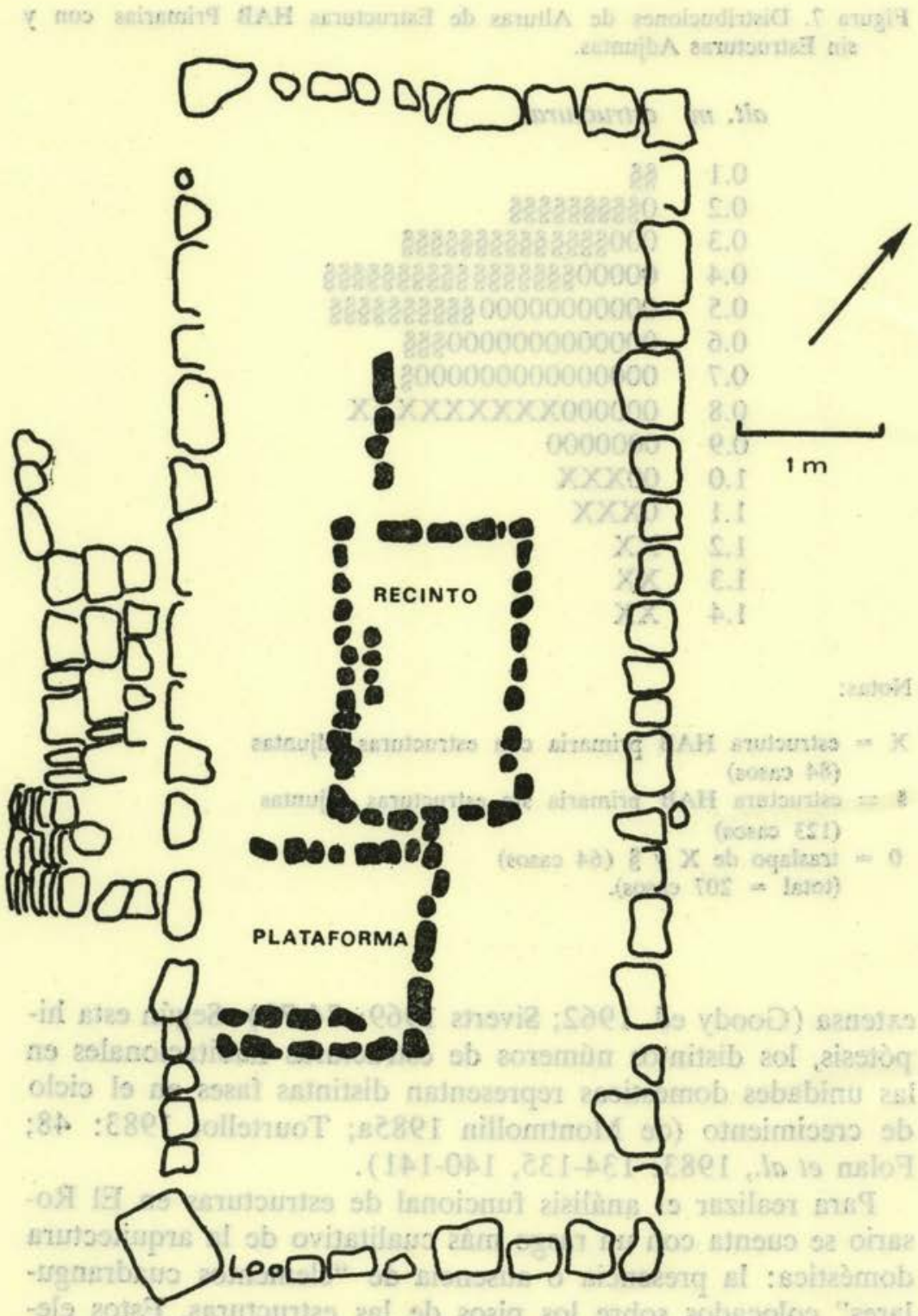

Figura 8. Elementos Cuadrangulares (Estructura 1, Sección A). 
Río Grijalva (Rivero 1977; Con Uribe 1981). En El Rosario mismo, se encontraron 20 elementos cuadrangulares dentro de 10 estructuras excavadas, y en el recorrido de superficie se registró la presencia (o la posible presencia) de elementos cuadrangulares en 182 estructuras. Hay varias clases de datos que dan apoyo a la idea de que la presencia de elementos cuadrangulares dentro de una estructura indica una función habitacional. 1. Los elementos cuadrangulares son muy comunes (en 182 estructuras) y según el "principio de la abundancia" (Haviland 1966) eso indicaría una asociación de tales elementos con actividades habitacionales. 2. Los elementos cuadrangulares se encuentran en toda la gama de tamaños de estructuras (cuadros 4 y 5) como fue el caso para las estructuras habitacionales en las dos hipótesis anteriores (ver las distribuciones de estructuras $\mathrm{HAB}$ de primer rango en figs. 4 y 5 y las distribuciones de estructuras $\mathrm{HAB}$ primarias en figs. 6 y 7 ).

\section{Cuadro 4}

Distribución de elementos cuadrangulares entre cuartiles de área

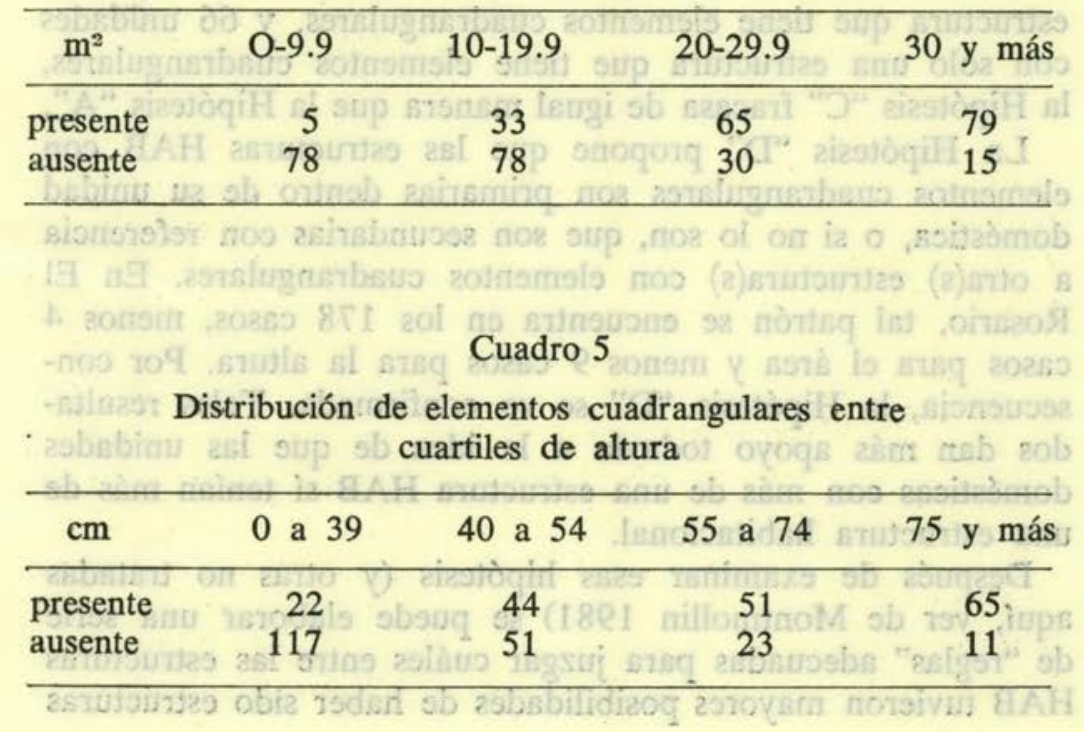


3. Aunque los elementos cuadrangulares (como plataformitas) se parecen a los pequeños altares que se encuentran al aire libre en algunas unidades domésticas, los elementos cuadrangulares excavados no mostraron señas de uso ritual especializado. 4. Muchas unidades domésticas tienen al menos una estructura con elemento cuadrangular (119 de los 163).

Es útil aceptar el supuesto de que la presencia de elementos cuadrangulares en una estructura indica un uso habitacional (y el supuesto más discutible de que la ausencia de tales elementos indica la posibilidad de un uso como estructura adjunta). Tales supuestos nos dan otro criterio funcional. Tener otro criterio es una ventaja porque diluye el excesivo (y doble) papel que tiene el área (de las plataformas), como criterio funcional (en la CFP) y como criterio de rango sociopolítico (en las Hipótesis "A" y "B").

Con los elementos cuadrangulares se pueden también utilizar procedimientos sencillos de evaluación de hipótesis. Por ejemplo, la Hipótesis " $C$ " propone que en cada unidad doméstica no había más de una estructura $\mathrm{HAB}$ con elementos cuadrangulares, y así, no más de una estructura habitacional. Este planteamiento se parece al de la Hipótesis "A". Dado que (entre las unidades domésticas con más de una estructura $\mathrm{HAB}$ ) se encuentran 53 unidades domésticas con más de una estructura que tiene elementos cuadrangulares, y 66 unidades con sólo una estructura que tiene elementos cuadrangulares, la Hipótesis "C" fracasa de igual manera que la Hipótesis "A".

$\mathrm{La}$ Hipótesis " $\mathrm{D}$ " propone que las estructuras $\mathrm{HAB}$ con elementos cuadrangulares son primarias dentro de su unidad doméstica, o si no lo son, que son secundarias con referencia a otra(s) estructura(s) con elementos cuadrangulares. En El Rosario, tal patrón se encuentra en los 178 casos, menos 4 casos para el área y menos 9 casos para la altura. Por consecuencia, la Hipótesis "D" se ve confirmada. Tales resultados dan más apoyo todavía a la idea de que las unidades domésticas con más de una estructura $\mathrm{HAB}$ sí tenían más de una estructura habitacional.

Después de examinar esas hipótesis (y otras no tratadas aquí, ver de Montmollin 1981) se puede elaborar una serie de "reglas" adecuadas para juzgar cuáles entre las estructuras $\mathrm{HAB}$ tuvieron mayores posibilidades de haber sido estructuras 
adjuntas. Sin entrar en detalles (ver de Montmollin 1981), resulta que el manejo de todas esas reglas indica que casi ninguna de las 300 estructuras $\mathrm{HAB}$ tiene que ser reclasificada como estructura adjunta. En otras palabras, la CFP es válida para El Rosario. Dado que el patrón de asentamiento en El Rosario es bastante típico con referencia a los otros sitios en el valle Santa Inés/El Rosario, se puede también concluir que la CFP es una tipología adecuada para otros sitios vecinos. Consecuentemente se ha podido utilizar la CFP para identificar a más de 4500 estructuras habitacionales dentro del señorío Rosario de la época del clásico tardío terminal (de Montmollin 1985a, 1985c). Obviamente, los detalles de esa tipología no van a servir en cada parte de la zona maya. En contraste con perspectivas anteriores que enfatizaban la homogeneidad del patrón de asentamiento dentro de la zona maya (Vogt 1961), ya se está llegando a entender que había una marcada variabilidad entre los patrones de asentamiento doméstico de las distintas regiones de la zona maya (Tourtellot 1983): Esto quiere decir que para cada región se necesita hacer estudios del tipo que se acaba de presentar aquí (o, mejor todavía, estudios más sofisticados y acabados) para identificar cuáles eran las estructuras habitacionales y cuáles las adjuntas.

Los resultados detallados de tales estudios tendrán que ser distintos sólo en parte por las diferencias actuales entre los patrones de asentamiento doméstico. También serán distintos por razón de los distintos supuestos y procedimientos analíticos utilizados por los investigadores. Con referencia a estas últimas diferencias, se puede tomar el ejemplo del área de las plataformas. En el análisis no se ha utilizado el supuesto de que tenía que haber una proporción constante de $10 \mathrm{~m}^{2}$ de piso por persona para fines habitacionales. Al contrario, se ha llegado a la conclusión de que algunas estructuras muy pequeñas (con áreas de $8-10 \mathrm{~m}^{2}$ ) probablemente sirvieron como viviendas para familias (sin pensar en un tamaño invariable para la familia más allá de un mínimo de dos adultos). Otro tipo de análisis se serviría del supuesto de que se necesita absolutamente $10 \mathrm{~m}^{2}$ por persona, tal vez fijándose en su aparente validez transcultural (Naroll 1962; Puleston 1973: 179-185). Combinado con el supuesto de que las familias deben tener un tamaño de entre 5 y 6 personas, un planteamiento de esta 
clase descartaría la posibilidad de encontrar estructuras habitacionales entre las estructuras de menos de $50 \mathrm{~m}^{2}$. Para El Rosario no se ha utilizado este segundo tipo de análisis por desconfiar de toda regla (o ley) de tipo transcultural, y porque no es muy útil para explorar la variabilidad entre las estructuras y unidades domésticas en $\mathrm{El}$ Rosario (recordándose que casi todas las estructuras tienen menos de $50 \mathrm{~m}^{2}$, con un promedio para las estructuras $\mathrm{HAB}$ de $25.6 \mathrm{~m}^{2}$ ). Frente a las varias posibilidades analíticas y teóricas, queda claro que se tendrá que pensar mucho más en cómo se puede utilizar datos arqueológicos (en este caso de superficie) para llegar a conclusiones bien fundadas acerca de la estructura sociopolítica de los mayas del clásico, siempre respetando la variabilidad entre las distintas regiones de la zona maya.

\section{Agradecimientos}

Quiero agradecer al Instituto Nacional de Antropología e Historia, que dio permiso para los trabajos de campo en El Rosario. También quiero agradecer a la Fundación Arqueológica del Nuevo Mundo por su apoyo y sobre todo a Pierre Agrinier, quien dirigió el proyecto $\mathrm{El}$ Rosario. En fin, quiero agradecer a Catherine Starr por su valiosa colaboración en el registro de datos arquitectónicos en $\mathrm{El}$ Rosario.

\section{BIBLIOGRAFIA}

\section{Agrinier, Pierre}

1979 Late Classic Elite Vs. Non-Elite Domestic Variations from the Tenam Rosario. Zone. Ponencia presentada en el XLIII Congreso Internacional de Americanistas, Vancouver.

1983 Tenam Rosario: una posible localización del clásico tardío terminal Maya desde el Usumacinta. En Antropología e historia de los Mixe-Zoques y Mayas; ha menaje a Frans Blom, editado por Lorenzo Ochoa y Thomas A. Lee, Jr., pp. 241-254. Universidad Nacional Autónoma de México, México.

1984 Densidad de población contemporánea y del clásico tardío terminal en el valle de Santa Inés-Rosario, 
Chiapas. En XVII Mesa Redonda. Tomo I: 423-430. Sociedad Mexicana de Antropología, México.

AsHMORE, Wendy, (editora)

1981 Lowland Maya Settlement Patterns. University of New Mexico Press, Albuquerque.

Blake, T. Michael

1984 La detección de la organización social utilizando la arquitectura doméstica. En XVII Mesa Redonda. Tomo I: 471-478. Sociedad Mexicana de Antropología, México.

Con Uribe, María J.

1981 Laguna Francesa. Colección Científica, Arqueología No. 100.

De Montmollin, Olivier

1979 a Informe de las excavaciones realizadas en El Rosario (Tr-142) en 1979. Manuscrito depositado en la Fundación Arqueológica del Nuevo Mundo, San Cristóbal.

1979 b Report on a Detailed Architectural Survey at El Rosario ( $\mathrm{Tr}-142), 1979$. Manuscrito depositado en la Fundación Arqueológica del Nuevo Mundo, San Cristóbal.

1981 Functional Classification of Domestic Structures Using Survey Information From the Terminal Classic Period Site of El Rosario, Chiapas, Mexico. Manuscrito depositado en la Fundación Arqueológica del Nuevo Mundo, San Cristóbal.

1984 Patrón de asentamiento y de comunidad en el sitio de El Rosario, Chiapas, México. En XVII Mesa Redonda, tomo I: 415-422. Sociedad Mexicana de Antropología, México.

1985 a Classic Maya Settlement and Politics in the Rosario Valley, Chiapas, México. Tesis de doctorado, University of Michigan.

1985 b Patrones de asentamiento y estructura política en el señorío El Rosario. Ponencia presentada en el Primer Coloquio Internacional de Mayistas, México.

1985 c Settlement Pattern Survey in the Rosario Valley, Chiapas, Mexico. Working Papers No. 41 (Centre of Latin American Studies, Cambridge).

Folan, William J., Ellen R. Kintz, y Laraine A. Fletcher

1983 Coba: A Classic Maya Metropolis. Academic. Press, xog ontibs New York. 
GooDY, JACK R. (editor)

1962 The Developmental Cycle in Domestic Groups. Cambridge University Press, Cambridge.

Haviland, William A.

1966 Maya Settlement Patterns: A Critical Review. Middle American Research Institute (Tulane) Publication 26: 21-47.

1981 Dower Houses and Minor Centers at Tikal Guatemala: An Investigation into the Identification of Valid Units in Settlement Hierarchies. En Lowland Maya Settlement Patterns, editado por Wendy Ashmore, pp. 89117. University of New Mexico Press, Albuquerque.

KURJACK, EDWARD B.

1974 Prehistoric Lowland Maya Community and Social Organization-A Case Study of Dzibilchaltun, Yucatan, Mexico. Middle American Research Institute (Tulane), Publication 38.

NAROLL, RAOUL

1962 Floor Area and Settlement Population. American Antiquity, 27: 587-589.

Puleston, Dennis E.

1973 Ancient Maya Settlement Patterns and Environment at Tikal, Guatemala: Implications for Subsistence Models. Tesis de doctorado, University of Pennsylvania.

RIVERo T., SONIA E.

1977 Los Cimientos: análisis del patrón de asentamiento. Tesis de maestría, Escuela Nacional de Antropología e Historia.

SANDERS, William T.

1974 Chiefdom to State: Political Evolution at Kaminaljuyu, Guatemala. En Reconstructing Complex Societies (Supplement to the Bulletin of the American Schools of Oriental Research No. 20), editado por Charlotte B. Moore, pp. 97-121. Massachusetts Institute of Technology Press, Cambridge.

Siverts, Henning

1969 Oxchuc, una tribu Maya del México. Instituto Indigenista Interamericano, México.

TOURTELLOT, GAIR

1983 An Assessment of Classic Maya Household Composition. En Prehistoric Settlement Patterns, editado por 
Evon Z. Vogt y Richard M. Leventhal, pp. 35-54. University of New Mexico Press, Albuquerque.

VogT, Evon Z.

1961 Some Aspects of Zinacantan Settlement Patterns and Social Organization, Estudios de Cultura Maya, I: 131146. 